\title{
Cinnamon Bioactives and their Impact on Poultry Nutrition and Meat Quality - Impact on Human Health
}

\author{
Areeba Yaqoob ${ }^{1 *}$, Poushmal Abdul Razzaq ${ }^{2}$, Sana Iqbal ${ }^{1}$, \\ Obaid-ul-Hassan ${ }^{1}$, Hassam Ishtiaq ${ }^{3}$, Sajjad Hussain ${ }^{3}$, Waheed Ahmad ${ }^{3}$, \\ Muhammad Rizwan ${ }^{4}$, Mariam Altaf ${ }^{3}$, Hafza Fasiha Zahid ${ }^{5}$ and Akhtar Ali ${ }^{5}$ \\ ${ }^{1}$ Department of Food Sciences, Government College University Faisalabad, Sahiwal \\ Campus, Pakistan \\ ${ }^{2}$ Department of Human Nutrition and Dietetics, Mirpur University of Science and \\ Technology, AJK, Pakistan \\ ${ }^{3}$ National Institute of Food Science and Technology, University of Agriculture \\ Faisalabad, Pakistan \\ ${ }^{4}$ Department of Food Science and Technology, University of Central Punjab, Lahore, \\ Pakistan \\ ${ }^{5}$ School of Agriculture and Food, The University of Melbourne, Australia \\ *Corresponding Author: Areeba Yaqoob, Department of Food Sciences, Government \\ College University Faisalabad, Sahiwal Campus, Pakistan.
}

Email: areebayaqoob96@gmail.com

\begin{abstract}
In recent decades, natural phytochemicals gained much attention due to antioxidant and antimicrobial potential. Previously, synthetic preservatives widely used in meat industry to control pathogenic bacterial and to inhibit the lipid peroxidation in order to extend the shelf life and to improve the acceptability of meat and meat products. Due to consumer's awareness and demand and ban on synthetic additives in Europe, demand of plant derived natural products increased. Cinnamon is a wonderful spice that has been used from ancient's time to improve food flavor and sensory and organoleptic characteristics. Due to antioxidant and anti-microbial behavior potential of cinnamon bioactives, the natural antioxidants have been found effective in extending the shelf life and to increase the acceptability of meat by the consumers. Due to non-toxic, natural, residue free and easy availability of natural products make them highly acceptable as food additive and preservative. Cinnamon have been reported precious spice due to various beneficial effects like increased digestive enzymes, appetizer, immune stimulant, antioxidant, anti-microbial and anti-viral activities. Cinnamon is most widely used spice in poultry industry due to its vital phytochemicals and safe use.
\end{abstract}

Keywords: Cinnamon; Phytochemicals; Polyphenols; Antioxidants; Nutrition; Meat Quality

\section{Introduction}

The demand of poultry meat and meat products has increased exponentially in recent years. It is estimated that the production of chicken meat will reach 130 million tons at the end of 2020 and it will become the most consumed meat in all over the world. The significance of poultry industry has been increased in the world.
The chicken meat production was 115 million tons in 2015 estimated by FAO (Food and Agriculture Organization) which contributed about $40 \%$ of the total meat production in the world [1]. The chicken and their products are the most potent protein source as compared to the other meats. The poultry industry provides healthier and most economical food rather than red meat and other protein sources in human nutrition. The poultry industry is one 
of the fastest growing industry in the world and has a substantial contribution to human nutrition and food security [2]. The poultry meat and egg industries are the largest agricultural commodities and most common animal food source in the world.

Meat and meat products contain various nutrient compositions, including high-quality protein content, essential amino acids, B-group vitamins, minerals, and other nutrients [3] ideal for the growth and propagation of meat spoilage micro-organisms and common foodborne pathogens. Atmospheric oxygen, temperature, moisture, light, endogenous enzyme activity, and growth of microorganisms determine the quality and shelf life of meat [4] of which the growth of micro-organisms is regarded so far, the most significant factor in maintaining the safety and quality of meat although deteriorations can occur without micro-organisms [5]. The major principle of meat quality control is to eliminate or reduce microbial deterioration by following Food safety objectives (FSO) and hazard analysis and critical control point (HACCP) systems. The spoilage of meat and meat products is associated with bacteria such as Salmonella spp., Campylobacter jejuni, Escherichia coli 0157:H7, Listeria monocytogenes, Clostridium spp, Pseudomonas, Acinetobacter, Brochothrix thermosphacta, Lactobacillus spp., Enterobacter, etc., as well as molds and yeasts, which can cause outbreaks which severely affect public health and the economy $[3,5]$.

The high use of synthetic additives in food has raised many carcinogenic and toxic problems. Colorants and flavor were found to cause cancer and lead to DNA damage. In addition, well-known food additives such as benzoates can initiate allergies such as erythrasma and asthma and are believed to result in brain damage. Due to the growing concerns regarding the food safety and harm of chemical and synthetic preservatives, natural antimicrobials have been the attractive alternative trend for the food market. Plant extracts, essential oils, peptides, vitamin $\mathrm{C}$ (ascorbic acid), vitamin E (tocopherols), and protein hydrolysates have been proposed to prevent oxidation in processed meat products [6].

Therefore, natural products have been studied to serve as alternatives to synthetic compounds with objective to provide safe and wholesome food to mankind. The natural antioxidants have been found effective in extending the shelf life and to increase the acceptability of meat by the consumers [7]. Due to non-toxic, natural, residue free and easy availability of natural products make them highly acceptable as food additive and preservative. Cinnamon have been reported due to various beneficial effects like increased digestive enzymes, appetizer, immune stimulant, antioxidant, antimicrobial and anti-viral activities. Cinnamon is most widely used spice in poultry industry due to its phytochemicals and safe use. The plant phytochemicals gaining popularity due to their anti-oxidant and anti-microbial activities [8,9].

\section{Cinnamon}

From several decades, cinnamon (one of the most widely used spices) have been used in folk medicine. Nowadays, it has appealed great attention from researchers as a natural product with numerous health benefits [10]. Cinnamon has great potential as a natural alternative medicine for the cure and inhibition of many serious diseases (Alzheimer's Parkinson's and Diabetes) and for its antiinflammatory and anti-proliferative activities. It is highlighted in several studies that in addition to cinnamaldehyde as a main constituent, cinnamon extracts contain different phenolic compounds [10]. These bioactive compounds are responsible for different biological activities like antioxidant, natural repellent, anti-microbial, anti-inflammatory and anti-proliferative activities [11].

\section{Phytochemistry of cinnamon}

Cinnamon contains a significant number of phytochemicals although in small quantities. These compounds have great potential as antioxidant, antimicrobial, anti-inflammatory and other important properties $[12,13]$. The volatile compounds (cinnamon essential oil - CNO) mainly extracted from leaves, bark, root, stem. It is reported that cinnamon parts are enriched with aldehydes, alkenes, esters, phenols, acids, monoterpenes, diterpenes, sesquiterpenes, benzopyrones, hydrocarbons and flavonoids which have great antioxidant, antimicrobial and anti-inflammatory potential. Cinnamaldehyde, cinnamic acid, cinnamate, eugenol and other phenolic compounds are major bioactive compounds in cinnamon which play a significant role in various biological activities $[14,15]$. Antimicrobial, antioxidant, and anti-inflammatory properties are reported in various studies. According to research by using LCMS, data has shown that cinnamon consisted on condensed tannins, proanthocyanidins (PAs) and epicatechin which have $26.8 \%$, $23.2 \%$ and $3.6 \%$ proportions respectively. It shows that cinnamon has high polyphenol contents [16]. There are two types of anthocyanidins present in cinnamon which are A and B procyanidins $[17,18]$. PAs are oligomeric and polymeric flavan-3-ols: a type of

Citation: Areeba Yaqoob., et al. "Cinnamon Bioactives and their Impact on Poultry Nutrition and Meat Quality - Impact on Human Health". Acta Scientific Nutritional Health 6.2 (2022): 29-38. 
tannin while procyanidin contains (+) - catechin and (-) epicatechin [19]. The composition of cinnamon has also been investigated by other research groups who found that cinnamon bark enclosed with high levels of tannins which were comprised of 3.6\% epicatechins and $23.2 \%$ PAs [16].

\section{Cinnamon and poultry}

Utilization of cinnamon in poultry feed

Cinnamon is used in poultry diet as natural feed additive. Due to their antioxidant and anti-microbial activities, the bioactive compounds cinnamaldehyde, phenolic compounds and others have been used in the feed of poultry to improve growth performance, health and meat quality, blood metabolites, carcass traits, immunity and blood metabolites [20].

\section{Feed intake (FI) and growth performance}

The optimum performance of poultry can be mapped by a range of parameters including FCR, apparent metabolizable energy (AME), residual FI (RFI), BWG, and time to gain ideal market weight. The maintenance of growing demand of poultry products is possible only with most efficient feed efficiency. Many studies had been conducted to check the potential impact of dietary supplementation of cinnamon (oil and powder) and their bioactive constituents as natural growth promotors (NGPs) in poultry industry. It is reported in recent studies that cinnamon powder, cinnamaldehyde or their essential oils alone or combination with other oils have positive impact on the production of poultry. The potential effects include improved growth performance, increased FI, and improved overall health performance and feed efficiency. Al-Kassie [21] conducted a research on broiler chicks for 42 days to investigate the potential impact of EO derived from cinnamon on BWG, FI, FCR and overall health performance of chickens. According to the results, broilers fed with 200 ppm CNO had higher BWG, FI and FCR as compared to the group fed with $100 \mathrm{ppm}$ CNO.

An experiment was conducted by Sadeghi and Moghaddam [22] to evaluate the effects of cinnamon powder on broiler (Cobb-500) growth performance and other parameters under heat stress. It was observed that birds fed with cinnamon supplemented diets had better growth performance in terms of BWG and reduction in the FCR [22]. In another study, the potential effect of cinnamon powders $(0.05 \%)$ on growth performance under heat stress was evaluated. It was reported that the feed supplemented with cinnamon powders has increased BWG and FI as compared to control group [23]. It was suggested that cinnamon alone or with other spices has great potential to improve growth performance of poultry birds. It was reported that the addition of cinnamon or cinnamon extract in broilers feed significantly improve the growth performance, BWG and less feed to gain ratio. The BWG was higher in broilers fed with $3.0 \%$ cinnamon powder as compared to control feeds. Toghyani., et al. [24] investigated that addition of $2 \%$ cinnamon powder significantly improve the BWG of broilers at 28 and 42 day as compared to $4 \%$ cinnamon powder. FCR was low as compared to control group birds at $28^{\text {th }}$ day. It was suggested that cinnamon could be used as an alternative natural growth promotor in broilers. Muhl and Liebert [25] concluded that two PFAs supplemented with 3\% cinnamaldehyde, $2 \%$ capsicum oleoresin, $5 \%$ carvacrol or the alkaloids sanguinarine and chelerythrin have no significant impact on growth parameters: BWG, FI and FCR in male broiler chickens. Ebrahimi., et al. [26] reported that inclusion of cinnamon in broilers (Cobb 500) diet improved the BWG on 28 day. FI and feed to gain ratio was significant in cinnamon fed broilers as compared to cumin and ginger. Sang-Oh., et al. [27] found that 5\% cinnamon powder has more potential to improve the growth performance in male broilers (Ross $\times$ Ross 380). They calculated BWG, FI and feed efficiency for the period of five weeks.

Some contradictory results have also been reported. In recent times, Rashid, Mirani, Zehra, Gilani, Ashraf, Azhar, Al-Ghanim, AlMisned, Al-Mulahim and Mahboob [2] reported that cinnamon based diet had significant impact on FCR, BWG and FI as compared to the control groups on 42 day [2]. The enhancing effect of CNO on the growth performance and feed efficiency was due to the increasing the secretion of endogenous enzymes, cultivating the intestinal ecosystem, improving the immune system through provoking the antimicrobial and antioxidant activities.

\section{Blood chemical profile}

Rashid, Mirani, Zehra, Gilani, Ashraf, Azhar, Al-Ghanim, AlMisned, Al-Mulahim and Mahboob [2] investigated that cinnamon based phytogenic feed additives help to reduce the cholesterol level in blood which is ultematly positively impact the human health. Reportedly, cinnamon based diet plays a significant role to reduce the cholesterol in blood profile which is highly required as consumer's demand in all over the world. It is highly important to note that cinnamon trials on poultry birds were not consistent for blood 
chemical profile and immune system responses. The response variations among poultry experimental trails related to health status of the bird. While, MR [28] documented that inclusion of cinnamon and turmeric in broilers diet had significant impact to reduce the heterophil to lymphocyte ratio which help to protect the liver from radical lethal agents as well as aid to stabilize the cell membrane [29].

Similar findings were reported by Najafi and Taherpour [30] who showed that cinnamon help to reduce the cholesterol level in poultry. In another study, Hossain., et al. [31] suggested that 1.0\% cinnamon powder had significant impact to increase the antibody SP ratio (ratio of sample and positive control) for Newcastle disease and lower the blood glucose level. Furthermore, inclusion of cinnamon and zinc in poultry diet exhibited positive impact on blood parameters under cold stress conditions [32]. Cinnamon bioactive components suppress the activity of $\beta$-hydroxy $\beta$-methylglutaryl coenzyme A which is considered a vital enzyme for the synthesis of cholesterol $[33,34]$. To sum up, the reduction of oxidative and free radicals by the action active compounds, ultimately diminishes the production of MDA.

\section{Meat quality}

Nowadays, consumers give preference to the meat and meat products which are grown on natural feed additives rather than antibiotics due to their health concerns. The essential oils are widely used to improve the meat quality due to its easier acceptance in the global market. The inclusion of CNO in poultry feed can influence the meat quality by changing the oxidative stability of meat or by modifying the fatty acid profile of meat and meat products [35]. The CNO can be specialized as natural green preservative to boost the meat quality and shelf life as well as to prevent meat from pathogenic microorganisms. A study was conducted to examine the impact of cinnamon powders on lipid peroxidation and meat quality of chicken under heat stress. The data indicated that cinnamon and other feed supplements compensated the dry matter (DM) and pH to some extent which was decreased due to heat stress but was unable to restore them up to the control groups. Additionally, free radical scavenging activity and the TBA reactive substances had significantly reduced in meat of broilers fed on cinnamon and turmeric as compared to other treatments. There was no significant difference between crude protein and ash contents of thigh meat on 42 day [36].
The high contents of polyunsaturated fatty acids in chicken meat may reduce the shelf life of meat and additionally cause off-flavours and off-odours. The synthetic preservatives have health concerns and due to the consumer demand about "natural or antibiotic free". The interest in the antioxidant and antimicrobial properties of CNO has been increased. Ciftci., et al. [37] investigated that inclusion of CNO in broilers diet reduced cholesterol levels and total saturated fatty acids (SFA) while increased total unsaturated fatty acids and $\omega-6$ fatty acids as compared to the control group. They concluded that CNO had antioxidant and hypocholesterolemic effect which ultimately improved quality of meat. Furthermore, Chowdhury., et al. [38] reported that supplementation of CNO had increased $\omega-3$ fatty acids as compared to the other groups.

The fatty acid profile of poultry meat can be modified by using cinnamon due to their antioxidant potential. For example, Crespo and Esteve-Garcia [39] reported that total PUFA increased in poultry meat fed on CNO. The meat with optimum concentration of $\omega-6$ and $\omega-3$ is beneficial for human health [38]. The strong antioxidant potential of CNO protects the PUFAs in chicken meat from lipid peroxidation [40]. In addition, Dalkilic., et al. [41] investigated that PUFAs, $\omega-3$ and $\omega-6$ levels were high while SFAs levels were low in CNO fed chicken meat. The antioxidant properties of cinnamon and other phytogenic extracts on chicken meat play a pivotal role for the retention of $\mathrm{CNO}$ in the meat which may contribute in the flavor and aroma of the cooked meat. Likewise, Jamroz., et al. [42] documented that supplementation of phytogenic extracts (cinnamaldehyde, capsaicin and carvacrol) enhanced meat tenderness, juiciness, smell, taste and color as compared to the control groups.

It is reported in recent studies that cinnamon powder, cinnamaldehyde or their essential oils alone or combination with other oils have potential to improve the chicken meat quality. Al-Kassie [21] reported that CNO reduced the abdominal fat in broiler meat. Almost similar findings were reported by Isabel and Santos [43] who reported that abdominal fat reduced while breast meat yield increased in CNO fed group. Furthermore, Sang-Oh, Chae-Min, Byung-Sung and Jong [27] showed that the water holding capacity was higher while TBARS was low in cinnamon fed group. Additionally, abdominal fat was lower and meat yield was higher in cinnamon group. Cinnamon fed samples improved the sensory attributes of broiler chicken. They concluded that cinnamon can improve the quality and shelf life of chicken meat. Interestingly, many studies have been documented about the positive impacts of EOs

Citation: Areeba Yaqoob., et al. "Cinnamon Bioactives and their Impact on Poultry Nutrition and Meat Quality - Impact on Human Health". Acta Scientific Nutritional Health 6.2 (2022): 29-38. 
to improve meat yield while the reduction in the abdominal fat of broiler meat [44]. Conversely, Sampath and Atapattu [45] reported that cinnamon powder had no significant effect on abdominal fat and meat yield. Safa Eltazi and Research [46] concluded that cinnamon improved the sensory attributes of the chicken meat. The cinnamon powder administration in broilers diet had significant impact on abdominal fat [47].

The sensory evaluation (taste and savor) of cinnamon fed chicken meat were significantly better than the control group. The given data illustrates that cinnamon bioactive constituents may be responsible to improve the sensory quality of chicken meat. In addition, Singh., et al. [48] documented that the supplementation of cinnamon in broilers diet enhanced the sensory characteristics of chicken meat as compared to the control treatments. To sum up, antioxidant potential of cinnamon to inhibit the lipid peroxidation in meat and meat products has more concern in poultry industry. Cinnamon plant oils have been used in poultry diet may readily shift the fatty acid profile and generally improve the PUFAs which cause susceptibility of oxidation in meat. Concomitantly, cinnamon has antioxidant compounds which improved the oxidative stability of chicken meat. The main antioxidant compounds are cinnamaldehyde, cinnamic acid, tocopherols, phenolic compounds and many other bioactive compounds. Their antioxidant capacity and radical scavenging properties have been considered pivotal for improving the quality and shelf life of chicken meat.

\begin{tabular}{|c|c|c|c|c|c|}
\hline Feed composition & Feed level & $\begin{array}{l}\text { Bird } \\
\text { type }\end{array}$ & $\begin{array}{l}\text { Period } \\
\text { (days) }\end{array}$ & Results & Ref. \\
\hline CNO & $250 \mathrm{mg} / \mathrm{kg}$ & Broiler & 35 & No effect on meat quality, decreased meat cholesterol level & [47] \\
\hline CNO & $500 \mathrm{mg} / \mathrm{kg}$ & Broiler & 38 & $\begin{array}{l}\text { Increased phagocytic activity, } \\
\text { reduced malondialdehyde level and ALT activity of plasma, increased } \\
\text { gluthathione peroxidase activity }\end{array}$ & \\
\hline CNO & $200 \mathrm{mg} / \mathrm{kg}$ & Broiler & 42 & $\begin{array}{c}\text { Improved blood hematology, Decreased cholesterol and abdominal } \\
\text { fat } \% \\
\text { Improved BWG and FCR }\end{array}$ & [21] \\
\hline $\mathrm{CNO}+\mathrm{CLO}$ & $100 \mathrm{ppm}$ & Broiler & 46 & Improved FCR and breast weight & [43] \\
\hline $\mathrm{CNO}$ & $1 \mathrm{~g} / \mathrm{kg}$ & Broiler & 35 & Improved sensory attributes of meat & {$[46]$} \\
\hline $\begin{array}{l}\text { Cinn. } \\
\text { hyde+Carv+Caps }\end{array}$ & $300 \mathrm{ppm}$ & Broiler & 48 & $\begin{array}{l}\text { Improved BWG and FCR, Improved sensory characteristics of meat, } \\
\text { higher breast muscle share }\end{array}$ & [42] \\
\hline
\end{tabular}

Table 1: Effect of cinnamon on nutrient digestibility, blood chemical profile, meat quality and growth performance.

$$
\text { CNO = Cinnamon essential oil. }
$$

\section{Cinnamon and poultry health perspective}

The multiple functions of the gut immune system (i.e. avoid detrimental responses to food antigens and commensal microbes, prevent pathogen invasion and translocation to extra intestinal sites and exploit beneficial effects of commensal microbes) relies on the mucosa to maintain immune homeostasis and protect against prolonged inflammation. In summary, maintaining gut immune homeostasis, while mounting protective immunity to pathogens is primarily achieved through: (1) limiting direct bacterial contact with the intestinal epithelium and (2) rapid detection and removal of pathogens that penetrate the epithelium.

All animals play host to entire communities of commensal and symbiotic microbes. In the intestine, there are estimates of up to 
1014 bacterial cells, which is around 100 times greater than, for example, host (human or pig) cells. Thus, the gut microbiome acts as an additional complex organ within the host, containing more than one million genes (for comparison, this is about 40-50 times the number in the entire chicken genome [49]. These microbes receive their nutrients from the host (or host's diet) and, in turn, contribute essential nutrients (see below) to the host, promote intestinal development, enhance gut integrity, provide competitive exclusion of pathogens, modulate the regulation and function of the host immune system, and sustain immune homeostasis by controlling physiological inflammation. In germ-free animals (e.g. mice), both the mucosal and systemic immune systems are poorly developed, and these animals do not generate normal oral tolerance to dietary proteins, but these features can be overcome/restored if these animals are subsequently colonized by a conventional or defined microbiota. This demonstrates a clear link between intestinal colonization by microbes and the development of a normally functioning immune system. In addition, it is demonstrated that giving chicks the gut contents of healthy adult chickens profoundly increased their resistance to Salmonella infection. This contributed to the 'competitive exclusion' phenomenon and highlighted the critical role of a 'normal' gut microbiota in preventing undesirable microbial colonization. Moreover, in humans, disturbance of the gut microbiome through, for example, oral antibiotic therapy is frequently associated with diarrhea (antibiotic associated diarrhea) typically caused by Clostridium difficile. However, despite being manipulable, studies in pigs have also shown that the microbiota is somewhat resilient to change. Whilst weaning of piglets significantly influenced the gut microbiota, the microbiota of piglets 4 weeks after weaning still showed greater similarity with former littermates than to their new pen mates after they were mixed at weaning. These results demonstrate that the microbiota acquired early in life may be a strong determinant of its later composition. Additionally, it has been demonstrated that microbiota and immune system development are influenced by farm-to-farm differences. Given that, globally, farms will experience a multitude of gut health scenarios (i.e. 'better' or 'worse'), this indicates that there are multiple microbiotas/microbiomes that are associated with favourable intestinal health. Thus, whilst these may have common features, it is unlikely that there is a single microbiota/microbiome that we are striving to achieve but rather a range of possibilities, which may be dependent on local conditions.
Host nutrition and endogenous secretions strongly influence the gut microbiota and (thus) immune system, while nutritional deficiencies have long been known to impair immune function. There have been a number of reviews that have discussed the influence of nutrition on host immune responses and the intestinal microbiota [49]. The dynamic interactions between the gut microbiota and the immune system mean that microbiota modifications probably contribute significantly to the reported effects of nutrition on host immunity. As mentioned, the gut microbiota derives nutrients from the host's diet or endogenous secretions, whilst enhancing the nutritional value of the diet through the synthesis of essential nutrients (e.g. vitamins) and the production of complimentary enzymes (e.g. non-starch polysaccharidases). Therefore, whether through direct effects on immune function, or influences on microbiota composition and activity, nutrition is an integral component of gut health.

There is also interest in exogenous enzymes (alter substrate availability and digesta viscosity), plant-derived compounds (antimicrobial and other potential features), organic acids including butyric acid to improve the gut structure and inhibit the pathogens which will help to improve the meat quality and ultimately human health. Other, potentially more novel, attempts to influence gut health through microbiota and/or immunity modulation include exogenous provision of AMPs, antibodies (e.g. egg yolk), PRR ligands, bacteriophages and vaccines. Antibodies and AMPs can help supplement those innately present in the gut environment and thus impede (undesirable) bacterial contact with the epithelium. Bacteriophages are specific viruses that infect and replicate in bacteria and can influence host immunity. Therefore, bacteriophages with appropriate antibacterial and immunomodulatory activity may represent an effective option to promote gut health. PRR ligands have the potential to be used to upregulate innate immune pathways, through binding to their respective receptor, and thus promote innate defences. Vaccines can obviously be used to modulate immunity and generate responses specific to the antigen challenge. Better understanding of gut health could lead to specific mucosal vaccines that help shape the most appropriate immune response.

Cinnamon is abundant with flavonols as reported in many studies. Flavonols have strong antioxidant and antimicrobial properties and inhibit the growth of pathogenic bacteria in the poultry 
gut. The Equol is a major isoflavone, derived from the isoflavonoid daidzein, that have the ability to inhibit the oxidative modification induced by reactive oxygen species (ROS) in poultry gut [49]. The Equol increases the antioxidant activity of enzymes by enhancing the total antioxidant capacity as well promotes the expression of antioxidant genes in the gut which ultimately protects intestinal epithelial cells from oxidative damage from ROS.

It is reported that cinnamon is loaded with flavonoids (anthocyanins and PAs) [10]. These flavonoids are act as major antimicrobial agents in poultry gut. Same thing was reported by Iqbal., et al. [49] that flavonoids are potential agents in poultry gut.

Overall Correlation of gut microbiota with poultry performance

The poultry growth performance predicts by calculating the various parameters such as BWG, FI, RFI, FCR, AME and time taken to gain market weight. The gut microbiota is a vital dynamic organ which plays a significant role in digestion and absorption of feed nutrients in the gut. The composition of gut microbiota sig- nificantly affects the FCR. In cecum studies, Faecalibacterium was associated with lower FCR while Lactobacillus were reported to increase the FCR. In another study, the presence of Campylobacter was reported negatively correlated with the growth performance by increasing FCR. Furthermore, E. coli, Leptotrichia, Pediococcus, Rohdococcus significantly reduced the FCR while Fructobacillus, Lactobacillus and Paralactobacillus were linked with increased FCR in the study of jejunum [49]. In the crop, Bifidobacterium, Lactobacillus, Enterobacteria, E. coli, Shigella and Actinobacteria have been reported to reduce the growth performance in terms of WBG while Faecalibacterium, Ruminococcus, Clostridium coccoides, Euryarchaeota and Bacteroidetes were associated with high productivity of poultry. In the ileum, some bacterial taxa have been found to be influenced the growth performance of chicken [49]. Generally, Lactobacillus species have been considered for the improvement of growth performance and better health in poultry birds. Some studies have been documented that Lactobacillus and Bacteroides in fecal samples are correlated with better growth performance. But, the exact relationship between the gut microflora and growth performance of poultry birds have not been yet fully decoded.

\begin{tabular}{|c|c|c|c|c|}
\hline Feed composition & Feed level & Gut microbiota & Gut part & Ref. \\
\hline CNO & $100 \mathrm{mg} / \mathrm{kg}$ & $\begin{array}{c}\text { Lactobacillus and Bifidobacterium } \uparrow \\
\text { E. coli } \downarrow\end{array}$ & Cecum & \\
\hline CNO & $200 \mathrm{mg} / \mathrm{kg}$ & Lactobacillus $\uparrow$ Coliforms count $\downarrow$ & Intestine & \\
\hline Cinnamon + other phytogenic feed additives & $10 \%$ cinnamon & $\begin{array}{c}\text { Total counts of Enterococcus spp. and } \\
\text { Lactobacillus spp. } \uparrow \text { Campylobacter spp. } \\
\text { and E. coli } \downarrow\end{array}$ & $\begin{array}{l}\text { Ileum and } \\
\text { cecum }\end{array}$ & [2] \\
\hline $\mathrm{CNO}+\mathrm{OA}$ & $500 \mathrm{mg} / \mathrm{kg}$ & $\begin{array}{l}\text { No effect on total bacterial counts, } E \text {. } \\
\text { coli and Lactobacillus, Clostridium and } \\
\text { Salmonella counts } \downarrow\end{array}$ & $\begin{array}{l}\text { Ileum and } \\
\text { cecum }\end{array}$ & \\
\hline CNO & $300 \mathrm{mg} / \mathrm{kg}$ & $\begin{array}{l}\text { No change in Lactobacillus spp., E. coli } \\
\text { and Clostridium spp. } \downarrow\end{array}$ & Cecum & [38] \\
\hline Cinnamaldehyde+thymol & $5 g+15 \mathrm{~g} /$ tonne & E. coli $\downarrow$ & Cecum & \\
\hline Cinnamaldehyde+carvacrol+capsaicin & $100 \mathrm{mg} / \mathrm{kg}$ & $\begin{array}{l}\text { Lactobacillus counts } \uparrow, \text { E. coli and } \\
\text { Clostridium perfringens } \downarrow\end{array}$ & $\begin{array}{l}\text { Ileum and } \\
\text { cecum }\end{array}$ & \\
\hline
\end{tabular}

Table 2: Effect of cinnamon on gut microbiota of chicken.

CNO = Cinnamon Essential Oil, OA = Oxalic Acid.

\section{Conclusion}

Cinnamon has significant potential due to their bioactive compounds especially volatile compounds and phenolic compounds.
Cinnamon additives can be used to extend the shelf life of meat as food preservative as well as to inhibit the pathogenic bacteria in gut and on meat. Cinnamon bioactives help to maintain gut health 
status which ultimately help to minimize the disease and pathogens which leads to improve the meat quality. Cinnamon bioactive metabolites help to reduce cholesterol in blood and meat which is beneficial for human health. Antioxidant compounds in meat can exert and help to improve human health status by improving the antioxidants in human body. Further research can be conducted to validate the results how antioxidants of cinnamon can be transferred to human body and their positive effects on human body organs.

\section{Author's Contribution}

Areeba Yaqoob wrote final manuscript. All other authors also contributed to make this article publishable.

\section{Conflict of Interest}

No

\section{Bibliography}

1. Ali A., et al. "Cinnamon: A natural feed additive for poultry health and production-a review". Animals (Basel) (2021): 11.

2. Rashid Z., et al. "Enhanced modulation of gut microbial dynamics affecting body weight in birds triggered by natural growth promoters administered in conventional feed". Saudi Journal of Biological Sciences 27.10 (2020): 2747-2755.

3. Pereira PM and Vicente AF. "Meat nutritional composition and nutritive role in the human diet". Meat Science 93 (2013): 586592.

4. Zhou GH., et al. "Preservation technologies for fresh meat - a review”. Meat Science 86 (2010): 119-128.

5. Lorenzo JM., et al. "Main groups of microorganisms of relevance for food safety and stability: General aspects and overall description". Innovative Technologies for Food Preservation (2018): 53-107.

6. Ji J., et al. "Essential oils as natural antimicrobials applied in meat and meat products-a review". Critical Reviews in Food Science and Nutrition (2021): 1-17.

7. Chou 0., et al. "Lc-esi-qtof-ms/ms characterisation of phenolics in herbal tea infusion and their antioxidant potential". Fermentation 7 (2021): 73.

8. Sharifi-Rad J., et al. "Lc-esi-qtof-ms/ms characterization of phenolic compounds from pyracantha coccinea m. Roem. And their antioxidant capacity". Molecular and Cellular Biology 67 (2021): 201-211.
9. Bashmil YM., et al. "Screening and characterization of phenolic compounds from australian grown bananas and their antioxidant capacity". Antioxidants 10 (2021): 1521.

10. Ali A., et al. "Comprehensive profiling of most widely used spices for their phenolic compounds through lc-esi-qtofms (2) and their antioxidant potential". Antioxidants (Basel) (2021): 10 .

11. Razzaq PA., et al. "A comprehensive review on antidiabetic properties of turmeric". Life Science Journal 17 (2020): 26-39.

12. Ribeiro-Santos R., et al. "Technology. Revisiting an ancient spice with medicinal purposes: Cinnamon". Trends in Food Science and Technology 62 (2017): 154-169.

13. Ali A., et al. "Lc-ms/ms-qtof screening and identification of phenolic compounds from australian grown herbs and their antioxidant potential". Antioxidants (Basel) 10 (2021): 1770.

14. Rao P and Gan S. "Cinnamon: A multifaceted medicinal plant. Eveidence-based complementary and alternative medicine". Hindawi Publishing Corporation (2014).

15. Namazi N., et al. "The impact of cinnamon on anthropometric indices and glycemic status in patients with type 2 diabetes: A systematic review and meta-analysis of clinical trials" 43 (2019): 92-101.

16. Shan B., et al. "Antibacterial properties and major bioactive components of cinnamon stick (cinnamomum burmannii): Activity against foodborne pathogenic bacteria". Journal of Agricultural and Food Chemistry 55 (2007): 5484-5490.

17. Jarvill-Taylor KJ., et al. "A hydroxychalcone derived from cinnamon functions as a mimetic for insulin in 3t3-l1 adipocytes". Journal of the American College of Nutrition 20 (2001): 327336.

18. Lu Z., et al. "Hypoglycemic activities of a-and b-type procyanidin oligomer-rich extracts from different cinnamon barks". Phytomedicine 18 (2011): 298-302.

19. Passos CP., et al. "Evidence for galloylated type-a procyanidins in grape seeds". Food Chemistry 105 (2007): 1457-1467.

20. Cottrell JJ., et al. "Recent advances in the use of phytochemicals to manage gastrointestinal oxidative stress in poultry and pigs". Animal Production Science 10 (2021): 1071.

21. Al-Kassie GAJPVJ. "Influence of two plant extracts derived from thyme and cinnamon on broiler performance". The Pakistan Veterinary Journal 29 (2009): 169-173. 
22. Sadeghi A., et al. "The effects of turmeric, cinnamon, ginger and garlic powder nutrition on antioxidant enzymes' status and hormones involved in energy metabolism of broilers during heat stress" 8 (2018): 125-130.

23. Kanani PB., et al. "Effects of cinnamon (cinnamomum zeylanicum) and turmeric (curcuma longa) powders on performance, enzyme activity, and blood parameters of broiler chickens under heat stress" 4 (2016): 47-53.

24. Toghyani M., et al. "Evaluation of cinnamon and garlic as antibiotic growth promoter substitutions on performance, immune responses, serum biochemical and haematological parameters in broiler chicks". Livestock Science 138 (2011): 167-173.

25. Muhl A., et al. "Growth, nutrient utilization and threonine requirement of growing chicken fed threonine limiting diets with commercial blends of phytogenic feed additives". The Journal of Poultry Science 44 (2007): 297-304.

26. Ebrahimi M., et al. "Effect of cinnamon, red pepper, ginger and cumin on broilers performance". Research Opinions in Animal and Veterinary 3 (2013): 131-135.

27. Sang-Oh P., et al. "The meat quality and growth performance in broiler chickens fed diet with cinnamon powder". Journal of Environmental Biology 34 (2013): 127.

28. MR AJPSJ. "Effects of dietary inclusion of turmeric (curcuma longa) and cinnamon (cinnamomum verum) powders on performance, organs relative weight and some immune system parameters in broiler chickens". Journal of Poultry Science 2 (2014): 153-163.

29. Singh G., et al. "A comparison of chemical, antioxidant and antimicrobial studies of cinnamon leaf and bark volatile oils, oleoresins and their constituents". Food and chemical toxicology: an international journal published for the British Industrial Biological Research Association 45 (2007): 1650-1661.

30. Najafi S and Taherpour KJJASA. "Effects of dietary ginger (zingiber ofjicinale), cinnamon (cinnamomum), synbiotic and antibiotic supplementation on performance of broilers". Journal of Animal Science Advances 4 (2014): 658-667.

31. Hossain M., et al. "Evaluation of locally available herbs and spices on physical, biochemical and economical parameters on broiler production". International Journal of Plant, Animal and Environmental Sciences 4 (2014): 317-323.
32. Torki M., et al. "Single and combined effects of zinc and cinnamon essential oil in diet on productive performance, egg quality traits, and blood parameters of laying hens reared under cold stress condition". International Journal of Biometeorology 59 (2015): 1169-1177.

33. Elson C., et al. "Impact of lemongrass oil, an essential oil, on serum cholesterol”. Lipids 24 (1989): 677-679.

34. Goldstein JL and Brown MSJN. "Regulation of the mevalonate pathway". Nature 343 (1990): 425-430.

35. Wenk CJAAJoAS. "Herbs and botanicals as feed additives in monogastric animals". Asian Australasian Journal of Animal Sciences 16 (2003): 282-289.

36. Kanani PB., et al. "In Effect of dietary turmeric and cinnamon powders on meat quality and lipid peroxidation of broiler chicken under heat stress condition". Veterinary Research Forum, Faculty of Veterinary Medicine, Urmia University, Urmia, Iran (2017): 163.

37. Ciftci M., et al. "Effects of dietary antibiotic and cinnamon oil supplementation on antioxidant enzyme activities, cholesterol levels and fatty acid compositions of serum and meat in broiler chickens". Acta Veterinaria Brno 79 (2010): 33-40.

38. Chowdhury S., et al. "Different essential oils in diets of broiler chickens: 2. Gut microbes and morphology, immune response, and some blood profile and antioxidant enzymes". Animal Feed Science and Technology 236 (2018): 39-47.

39. Crespo N and Esteve-Garcia EJPS. "Dietary fatty acid profile modifies abdominal fat deposition in broiler chickens". Poultry Science 80 (2001): 71-78.

40. Botsoglou N., et al. "Effect of dietary oregano essential oil on performance of chickens and on iron-induced lipid oxidation of breast, thigh and abdominal fat tissues". British Poultry Science 43 (2002): 223-230.

41. Dalkilic B., et al. "Influence of dietary cinnamon oil supplementation on fatty acid composition of liver and abdominal fat in broiler chicken". Journal of Applied Animal Research 35 (2009): 173-176.

42. Jamroz D., et al. "The influence of phytogenic extracts on performance, nutrient digestibility, carcass characteristics, and gut microbial status in broiler chickens". Journal of Animal and Feed Sciences 12 (2003): 583-596. 
43. Isabel B and Santos YJJOAPR. "Effects of dietary organic acids and essential oils on growth performance and carcass characteristics of broiler chickens". Journal of Applied Poultry Research 18 (2009): 472-476.

44. Cross DE., et al. "Herbs, thyme essential oil and condensed tannin extracts as dietary supplements for broilers, and their effects on performance, digestibility, volatile fatty acids and organoleptic properties". British Poultry Science 52 (2011): 227-237.

45. Sampath H and Atapattu N. "Effects of cinnamon (cinnamomum zeylanicum) bark powder on growth performance, carcass fat and serum cholesterol levels of broiler chicken" (2013).

46. Safa Eltazi MJIJOIA and Research B. "Effect of using cinnamon powder as natural feed additive on performance and carcass quality of broiler chickens". International Journal of Livestock Research 2 (2014): 1-8.

47. Shirzadegan KJIJOAAS. "Reactions of modern broiler chickens to administration of cinnamon powder in the diet" 4 (2014): 367-371.

48. Singh J., et al. "Effect of cinnamon (cinnamomum cassia) powder as a phytobiotic growth promoter in commercial broiler chickens". Animal Nutrition and Feed Technology 14 (2014): 471-479.

49. Iqbal Y., et al. "Gut microbiota-polyphenol interactions in chicken: A review”. Animals (Basel) 10 (2020): 1391.

\section{Assets from publication with us}

- Prompt Acknowledgement after receiving the article

- Thorough Double blinded peer review

- Rapid Publication

- Issue of Publication Certificate

- High visibility of your Published work

Website: www.actascientific.com/

Submit Article: www.actascientific.com/submission.php

Email us: editor@actascientific.com

Contact us: +919182824667 